Thomas Gudermann • Veit Flockerzi

\title{
TRP channels as new pharmacological targets
}

Published online: 9 March 2005

C) Springer-Verlag 2005

Changes in intracellular ion concentrations are universal triggers of cell activation. $\mathrm{Ca}^{2+}$, for instance, is one of the most versatile intracellular second messengers in eukaryotic cells involved in a wide range of cellular processes including exocytosis, contraction, cell proliferation, development and apoptosis. Stimulation of target cells with neurotransmitters, hormones or growth factors leads to an elevation of the intracellular $\mathrm{Ca}^{2+}$ concentration brought about by release from intracellular stores and influx via $\mathrm{Ca}^{2+}$-permeable cation channels in the cell membrane (Clapham et al. 2001).

While excitable cells like neurons are well equipped with voltage-gated ion channels mediating the bulk of cation influx, the molecular identity of cation entry channels in non-excitable cells has been completely mysterious for quite some time. A major leap forward in our understanding of agonist-triggered cation influx was achieved by the discovery of Drosophila melanogaster visual transduction channels associated with the transient receptor potential (trp) and the trp-like (trpl) mutant phenotypes. Since then a new mammalian gene family of TRP homologs has been identified by homology screening (Montell et al. 2002; Clapham et al. 2003).

Based on structural homology and on systematic glycosylation scanning, TRP proteins are allocated to the structural superfamily of six-transmembrane spanning ion

This revised version was published online in June 2005 with corrections to the Reference list

T. Gudermann $(\bowtie)$

Institut für Pharmakologie und Toxikologie,

Fachbereich Medizin, Philipps-Universität Marburg,

Karl-von-Frisch-Str. 1,

35043 Marburg, Germany

e-mail: guderman@staff.uni-marburg.de

Tel.: +49-6421-2865000

Fax: +49-6421-2865600

V. Flockerzi

Institut für Experimentelle und Klinische Pharmakologie und Toxikologie, Universität des Saarlandes,

66421 Homburg, Germany channels like voltage-gated $\mathrm{K}^{+}$channels, the cyclic nucleotide-gated channel family, and single cassettes of voltage-activated $\mathrm{Ca}^{2+}$ and $\mathrm{Na}^{+}$channels (Fig. 1). Both $\mathrm{N}$ - and C-termini of TRP proteins are assumed to be localised intracellularly, and a channel pore loop appears to be bordered by transmembrane domains 5 and 6 . In specialised cells, some members of the TRP family participate in sensory processes like vision and hearing as well as temperature, pain, gustatory and pheromone perception. In addition, many TRP proteins are involved in ubiquitous, very basic cellular processes like sensing of osmotic or oxidative stress and mediating agonist-induced cation entry (Clapham 2003).

Based on primary amino acid homology, the conventional TRP proteins can be classified into three subfamilies: TRPC, TRPV, and TRPM (see Fig. 1). Three additional, more distantly related subfamilies have recently been defined: ANKTM1, a $\mathrm{Ca}^{2+}$-permeant, non-selective cation channel, is the only mammalian member of the TRPA branch of TRP proteins. It is activated by noxious cold temperature, pungent mustard oil compounds and may additionally serve as a mechanosensitive transduction channel in auditory hair cells as well as an ionotropic cannabinoid receptor. The three mucolipins, TRPML1, 2, and 3, appear to be ion channels in intracellular vesicles. Mutations in TRPML1 cause mucolipidosis type IV, a neurodegenerative lysosomal storage disorder, while genetic defects in TRPP2, a member of the TRPP subfamily, are frequently encountered in patients suffering from autosomal dominant polycystic kidney disease. The current compilation of review articles covers salient features of TRPC, TRPV, TRPM, and TRPP proteins at the cutting edge of science. In particular, the authors continue to explore the pharmacotherapeutic potential of this new class of ion channels, a fruitful endeavor that was started at the 25th Symposium at Rauischholzhausen Castle on "TRP channels as Pharmacological Targets" which took place on June 10-12, 2004.

TRPC proteins all share a common gating mechanism that involves the activation of phospholipase $\mathrm{C}$ isoforms and thus constitute prime molecular candidates responsible for phospholipase C-dependent cation influx. The molec- 
Fig. 1 Phylogenetic relationship and topology (inset) of mammalian TRP proteins
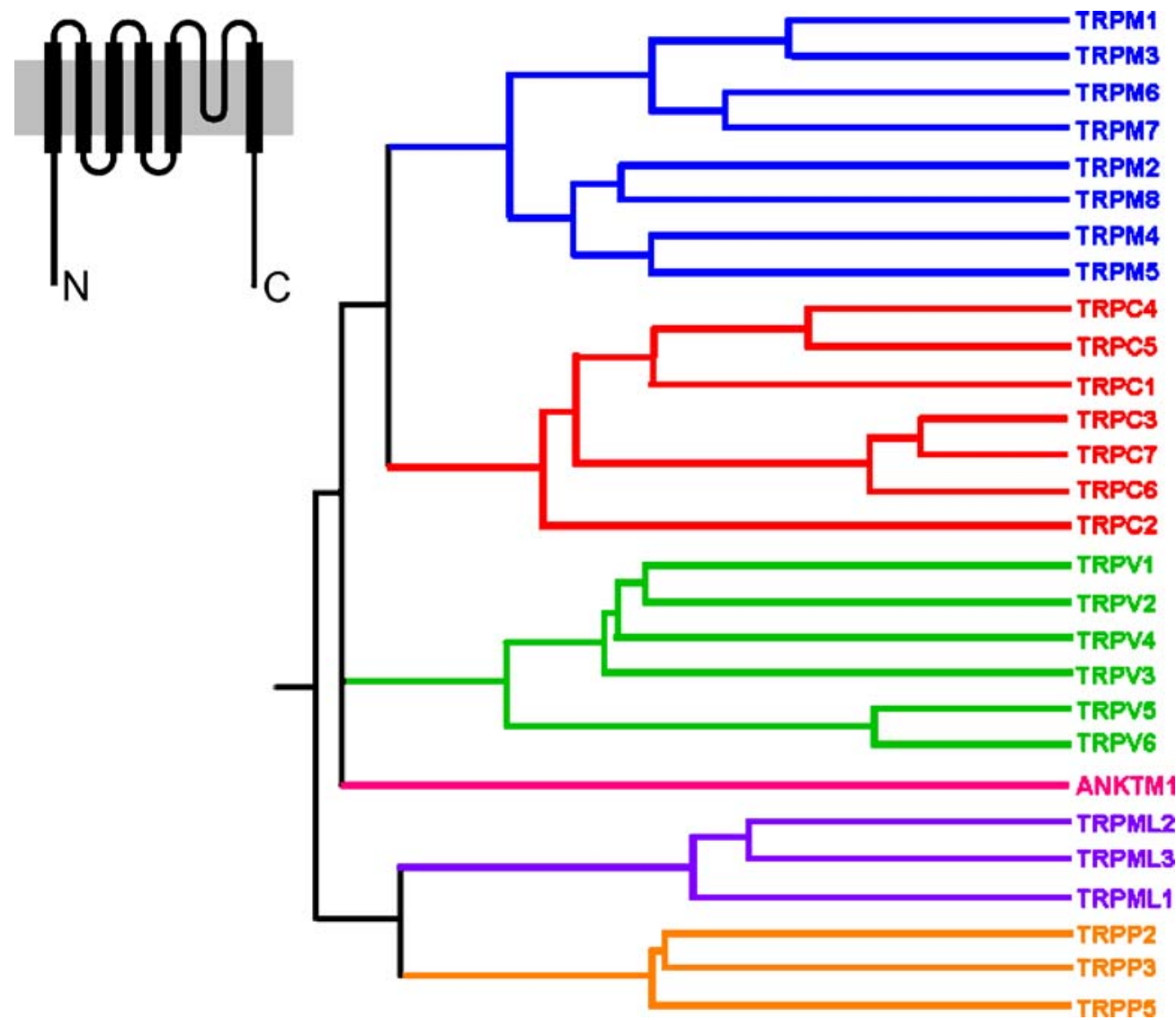

ular mechanism of phospholipase C-triggered TRPC channel activation, however, is still a moot issue. Towards this end, significant progress has recently been made in a first physiological system. TRPC2 which is specifically expressed in vomeronasal neurons is gated by diacylglycerols (Zufall 2005). TRPC2-deficient mice display severe deficits in the detection of pheromonal signals by the vomeronasal organ and are characterised by abnormal mating behaviour. On the basis of recent experimental results summarized by Frank Zufall in this issue, a clear physiological function can now be assigned to TRPC2 both at the cellular and at the systems level. In terms of its activation by diacylglycerols TRPC2 may serve as a paradigm for other TRPC channels expressed in their physiological environment.

For nearly all TRPC members, $\mathrm{Ca}^{2+}$ store-dependent and -independent activation mechanisms have been proposed. To further add to the complexity, several TRP proteins respond to a variety of stimuli acting from within and outside the cell. Thus, gating versatility is an emerging feature of TRP channels. In their contribution, Klaus Groschner and Christian Rosker focus on TRPC3, one of the most intensively studied TRPC protein (Groschner and Rosker 2005). TRPC3 is envisioned as a multifunctional signal transduction molecule physically and functionally embedded in cellular signalling networks. The proposed existence of multiple distinct TRPC3-containing signaling modules may lay the conceptual foundation for selective pharmacological targeting.
Among the TRPC subfamily, TRPC3, 6, and 7 share more than $70 \%$ identical amino acid residues and are also functionally related in that they are gated by diacylglycerols in a membrane-delimited manner, independent of protein kinase $\mathrm{C}$, when investigated in heterologous expression systems. Alexander Dietrich and colleagues provide a concise summary on the composition and functional properties of TRPC 3,6 , and 7 containing channel complexes and draw first conclusions on physiological functions derived from isolated tissues with downregulated channel activity and gene-deficient mouse models (Dietrich et al. 2005). Recent findings suggest that TRPC 3,6 , and 7 are not simply functionally redundant gene products, but have unique and indispensable roles in the control of airway and vascular smooth muscle tone.

Although there are a large number of publications on TRPC1, it is still unclear at present whether this channel subunit can form homomeric functional ion channels or whether functional channels require heteromultimerisation with other TRPC isoforms. TRPC4 and 5 have been identified as heteromeric partners of TRPC1 in vitro and in living cells. Of note, in different studies TRPC4 and TRPC 5 have been described as store-operated $\mathrm{Ca}^{2+}$-permeant channels or as non-selective cation channels activated in a phospholipase $\mathrm{C}$-dependent manner independent of intracellular $\mathrm{Ca}^{2+}$ store depletion. Tim Plant and Michael Schaefer interpret the available data on the regulation and permeability properties of TRPC4 and 5 and provide in- 
triguing explanations for the major functional discrepancies reported by different researchers (Plant and Schaefer 2005).

TRP proteins of the TRPC, TRPV, and TRPM families are expressed in lung tissues. Because cation channels are of key importance for the regulation of bronchoconstriction in response to various spasmogens, TRP channels may represent plausible molecular targets. As pointed out by $\mathrm{Su}$ $\mathrm{Li}$ and colleagues, respiratory disease is increasing in prevalence worldwide and physicians would certainly not mind further optimisation of current therapies (Gosling et al. 2005). Pending a detailed delineation of the physiological role of TRP channels in airway smooth muscle, these cation channels evolve into attractive drug targets for the treatment of lung diseases such as asthma, chronic obstructive pulmonary disease and pulmonary hypertension.

Compared to most TRPC and TRPM proteins, the physiological role of TRPV family members is fairly well understood. The latter ion channels are involved in sensory processes and in the absorption of $\mathrm{Ca}^{2+}$ in the gut and kidney. In analogy to the situation with other hexahelical cation channels, four TRP proteins assemble to form a functional channel complex. However, the structural basis governing the assembly of tetrameric complexes are only poorly understood and results from different groups are remarkably controversial. Barbara Niemeyer contributes an insightful and detailed review article on the structurefunction relationship mainly of TRPV channels (Niemeyer 2005). Particular emphasis is placed on the delineation of assembly signals of TRPV channels, particularly addressing the role of ankyrin repeats for channel complex formation. At present it is not known whether structural components such as tetramerisation domains are well conserved and to what extent conclusions on structure-function relationships can be extended from one distinct TRP channel to other family members.

Four of the six TRPV proteins are temperature-activated. In addition, TRPV1 is also activated by vanilloid compounds, the endogenous cannabinoid anandamide as well as by 12-lipoxygenase metabolites of arachidonic acid. In the case of TRPV4, gating versatility is exemplified quite impressively. The channel is activated by numerous physical and chemical stimuli including cell swelling, heat, phorbol esters, and also by epoxyeicosatrienoic acids. TRPV5 and TRPV6 have been isolated from $\mathrm{Ca}^{2+}$-transporting epithelia and play a major role in the intestinal uptake and renal reabsorption of $\mathrm{Ca}^{2+}$ as highlighted by René Bindels and colleagues (van Abel et al. 2005) summarizing the hormonal regulation, interaction with accessory proteins and pathophysiological relevance of the two closely related proteins. Both channels show strongly inwardly rectifying currents and are the most $\mathrm{Ca}^{2+}$-selective TRP channels. Interestingly, TRPV6 was also reported to exhibit salient features of $\mathrm{Ca}^{2+}$ release-activated $\mathrm{Ca}^{2+}$ (CRAC) channels when expressed in vitro.

Currently, the physiological role and the cellular regulation of several TRPM proteins are considerably less understood when compared with their TRPC and TRPV relatives. TRPM proteins display the structural hallmark of exceptionally long intracellular $\mathrm{N}$ - and C-termini. TRPM1 or melastatin, the founding member of this TRP subfamily, detected as a presumptive tumor suppressor in malignant melanoma cells, is a prominent example for a TRP protein whose cellular role and mechanism of action have remained utterly mysterious up to now. Christian Harteneck sums up the known functions and gating mechanisms of TRPM channels and provides a comprehensive overview on known pharmacological tools to probe TRPM channel function (Harteneck 2005).

A principally unsolved problem when trying to investigate TRP channels in their native environment is to unequivocally ascribe agonist-activated cation currents to molecularly defined TRP proteins. One reason for this awkward situation is our ignorance about the native composition of functional TRP channel complexes as alluded to earlier. An additional layer of complexity is introduced by transcriptional regulation and posttranscriptional RNA processing which may result in TRP channel variants with altered functional properties. Stefan Philipp and colleagues underscore the emerging relevance of transcriptional regulation and further processing of TRP channels and point to the daunting task of tackling the TRP transcriptome in order to gain further insight into the molecular mechanisms underlying the functional variability of TRP channels (Lis et al 2005).

Within the TRPM subfamily, TRPM2 is most probably one of the most thoroughly understood ion channels intimately involved in the activation process of neutrophil granulocytes. TRPM2 is one of three members of the TRPM subfamily that are set apart from other known ion channels because they harbour enzyme domains in their respective $\mathrm{C}$-termini and thus represent prototypes of chanzymes, an intriguing new protein family of enzymecoupled ion channels. TRPM2 is gated by binding of intracellular ADP ribose to its C-terminal ADP-pyrophosphatase domain. Andreas Lückhoff and colleagues point out that the chemoattractant peptide $N$-formyl-methionylleucyl-phenylalanin (fMLP) induces relevant increases in the ADP ribose concentration in neutrophils entailing TRPM2 activation. The signal transduction sequence from fMLP binding to its G-protein-coupled receptor up to TRPM2 gating offers several molecular targets whose pharmacological suitability is systematically discussed to modulate granulocyte function in inflammation.

Apart from TRPM2, the closely related TRPM6 and TRPM7 were also coined chanzymes because they contain C-terminal protein kinase domains which bear sequence similarity to elongation factor 2 serine/threonine kinases and other proteins also containing an $\alpha$-kinase domain. Whereas electrophysiological and biochemical analyses identified TRPM7 whose genetic inactivation is incompatible with life, as an important player in cellular magnesium homeostasis, the critical role of TRPM6 for epithelial magnesium transport emerged from the discovery of lossof-function mutations in patients with a severe form of hereditary hypomagnesemia called primary hypomagnesemia with secondary hypocalcemia. Vladimir Chubanov and colleagues summarize recent findings obtained from 
molecular genetic, biochemical, and electrophysiological studies with a special focus on the composition and (patho) physiological relevance of TRPM6/TRPM7 containing signalling complexes.

Among the two proteins PKD1 and PKD2 (TRPP2) which are found mutated in the vast majority of patients with autosomal dominant polycystic kidney disease, the latter one, TRPP2, is a six-transmembrane spanning $\mathrm{Ca}^{2+}$ permeable cation channel. However, similar to the situation with other TRP proteins, the cellular localisation of TRPP2 is a controversially debated issue. Ralph Witzgall presents a systematic overview of arguments against or in favour of a plasma membrane or intracellular localisation of TRPP2 and describes novel approaches to identify components of the trafficking machinery of TRPP 2 . These findings could serve as a model for other TRP proteins whose trafficking and physiologically relevant localisation are still discussed controversially.

Ion channels are one of only a limited number of protein classes apart from G-protein-coupled and nuclear hormone receptors and enzymes, that have been amenable to small-molecule drug discovery. The current review series illustrates that targeting TRP channels may allow the development of new drugs for diseases such as cancer and immune disorders, thereby expanding the traditional set of indications for the design of ion channel modulators. Although we are only just beginning to fully appreciate the physiological and pathophysiological role of TRP channels our knowledge is advancing at a quick pace.

So, stay tuned for you will definitely hear more about the exciting pharmacological prospects of this new family of ion channels.

\section{References}

Chubanov V, Mederos y Schnitzler M, Wäring J, Plank A, Gudermann T (2005) Emerging roles of TRPM6/TRPM7 channel kinase signal transduction complexes. Naunyn-Schmiedeberg's Arch Pharmacol 371:334-341
Clapham DE (2003) TRP channels as cellular sensors. Nature 426:517-524

Clapham DE, Runnels LW, Strübing C (2001) The TRP ion channel family. Nat Neurosci 2:387-396

Clapham DE, Montell C, Schultz G, Julius D (2003) International Union of Pharmacology. XLIII. Compendium of voltage-gated ion channels: transient receptor potential channels. Pharmacol Rev 55:591-596

Dietrich A, Mederos y Schnitzler M, Kalwa H, Storch U, Gudermann T (2005) Functional characterization and physiological relevance of the TRPC3/6/7 subfamily of cation channels. Naunyn-Schmiedeberg's Arch Pharmacol 371:257-265

Gosling M, Poll C, Li S (2005) TRP channels in airway smooth muscle as therapeutic targets. Naunyn-Schmiedeberg's Arch Pharmacol 371:277-284

Groschner K, Rosker C (2005) TRPC3: a versatile transducer molecule that serves integration and diversification of cellular signals. Naunyn-Schmiedeberg's Arch Pharmacol 371:251-256

Harteneck C (2005) Function and pharmacology of TRPM cation channels. Naunyn-Schmiedeberg's Arch Pharmacol 371:307 $-314$

Heiner I, Radukina N, Eisfeld J, Kühn F, Lückhoff A (2005) Regulation of TRPM2 channels in neutrophil granulocytes by ADP-ribose: a promising pharmacological target. NaunynSchmiedeberg's Arch Pharmacol 371:325-333

Lis A, Wissenbach U, Philipp S (2005) Transcriptional regulation and processing increase the functional variability of TRP channels. Naunyn-Schmiedeberg's Arch Pharmacol 371:315-324

Montell C, Birnbaumer L, Flockerzi V (2002) The TRP channels, remarkable functional family. Cell 108:595-598

Niemeyer B (2005) Structure-function analysis of TRPV channels. Naunyn-Schmiedeberg's Arch Pharmacol 371:285-294

Plant TD, Schaefer M (2005) Receptor-operated cation channels formed by TRPC4 and TRPC5. Naunyn-Schmiedeberg's Arch Pharmacol 371:266-276

van Abel M, Hoenderop JGJ, Bindels RJM (2005) The epithelial calcium channels TRPV5 and TRPV6: regulation and implications for disease. Naunyn Schmiedeberg's Arch Pharmacol 371: 295-306

Witzgall R (2005) Polycystin-2 - an intracellular or plasma membrane channel? Naunyn-Schmiedeberg's Arch Pharmacol 371: 342-347

Zufall F (2005) The TRPC2 ion channel and pheromone sensing in the accessory olfactory system. Naunyn-Schmiedeberg's Arch Pharmacol 371:245-250 